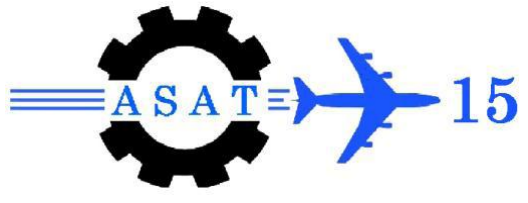

\title{
Modified Analysis of LSD in Laser Propulsion and Performance Prediction
}

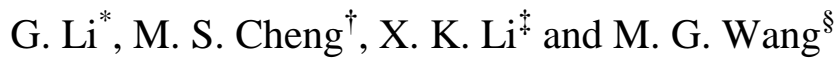

\begin{abstract}
Laser propulsion is one of the promising solutions to the cost morass of chemical rockets. Laser supported detonation (LSD) is an important phenomenon formed in laser radiation, which would significantly enhance the specific impulse with an appropriate laser intensity. When the intensity is high enough, the total number of particles is evidently different through the LSD wave, and the dissociation and ionization energy must be considered. Taking account of these impacts in the conservations of mass, momentum and energy, a modified analysis of LSD is presented and the flow state behind the LSD wave is obtained. Calculated results are compared with the classical Raizer's model and experimental data. Furthermore, performances of laser propulsion are evaluated based on a 1-D assumption. The study concentrates on a better understanding of LSD and laser propulsion.
\end{abstract}

Keywords: Laser propulsion, LSD, plasma, energy conversion, performance prediction

\section{Introduction}

Laser-supported detonation (LSD) is one of the most important phenomena of the interaction between laser and material, which is employed in many domains, such as laser propulsion, pulsed laser deposition of thin film, laser machining and so on. The fundament and application of LSD have attracted a large number of researchers' attention. For laser propulsion ${ }^{[1]}$, LSD is a significant mechanism to accelerate the propellant and improve the specific impulse.

Raizer taken LSD wave as hydrodynamic discontinuity, and assumed that the laser energy was completely absorbed in the region ${ }^{[2]}$. By counting the conservations of mass, momentum and energy, using the Jouguet condition, he deduced the relationship between LSD wave parameters and laser parameters. Based on Raizer's theory, Pirri ${ }^{[3]}$ and Reilly ${ }^{[4]}$ analyzed the performance of laser propulsion with one-dimensional and two-dimensional simplifications.

The biggest deficiency of these works is that they do not consider the effect of dissociation and ionization occurred in the propagation of LSD wave, which makes their results larger than the experimental data. Shimamura et al. ${ }^{[5]}$ measured the internal structure and electron density distribution by two-wavelength Mach-Zehnder interferometer.

\footnotetext{
Ph.D. Student, College of Aerospace Science and Engineering, National University of Defense Technology, Changsha, 410073, China. ligan0001@gmail.com

$\dagger \quad$ Professor, National University of Defense Technology, chengmousen@vip.sina.com

$\ddagger \quad$ Lecturer, National University of Defense Technology, 1xk0330@gmail.com

$\S \quad$ Lecturer, National University of Defense Technology, tlothf_18@sina.cn
} 
The results showed that, when the laser intensity was $50 \mathrm{MW} / \mathrm{cm}^{2}$, the electron density could rise up to $2 \times 10^{24} \mathrm{~m}^{-3}$ in $1 \mathrm{~atm}$ atmosphere and the ionization rate was about $4 \%$. If the LSD wave velocity was $10^{3} \mathrm{~m} / \mathrm{s}$, dissociation and ionization energy accounted for $12 \%$ of the total incident energy of the laser pulse. It is obvious that dissociation and ionization plays an important role in the conversion of laser energy, especially when the laser intensity is larger. For the intensity usually used in laser propulsion is in the range of $10^{7}$ and $10^{9} \mathrm{~W} / \mathrm{cm}^{2}$, dissociation and ionization have great impact on the propulsion performance. It is necessary to modify Raizer's model, discuss deeply, in order to obtain a more accurate understanding.

By considering the effect of dissociation and ionization in mass, momentum and energy conservation, a modified analysis of LSD was presented in this paper. Based on the analysis, the flow state parameters behind the LSD wave were obtained and compared with Raizer's model and the experimental data. Furthermore, adopting one-dimensional assumption, the propulsion performance of laser propulsion was predicted.

\section{Modified Model of LSD}

It is assumed that the LSD wave is a strong hydrodynamic discontinuity which moves supersonically and has no thickness. The incident laser is completely absorbed in the small region of the gap. In the inertial coordinate system fixed at the LSD wave front, a control volume can be established to deduce the relationship of the parameters flanked the LSD wave. The control volume model is shown in Fig.1, the subscript 1 and 2 denote front and back of LSD respectively.

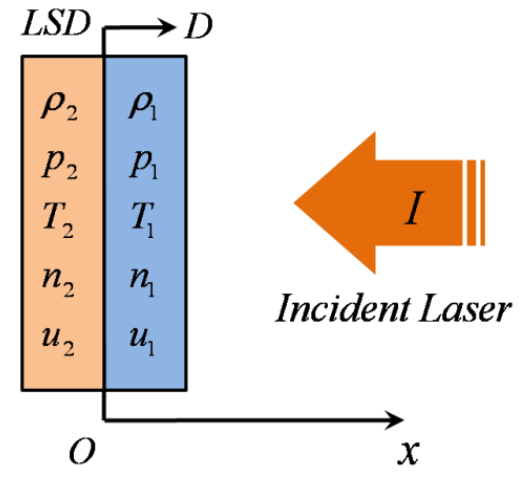

Fig 1 Control volume model of LSD.

The mass, momentum and energy conservation in the control volume are of the form

$$
\begin{gathered}
\rho_{1}\left(u_{1 n}-D\right)=\rho_{2}\left(u_{2 n}-D\right) \\
p_{1}+\rho_{1}\left(u_{1 n}-D\right)^{2}=p_{2}+\rho_{2}\left(u_{2 n}-D\right)^{2} \\
{\left[e_{2}+\frac{\left(u_{2 n}-D\right)^{2}}{2}+\frac{p_{2}}{\rho_{2}}\right]-\left[e_{1}+\frac{\left(u_{1 n}-D\right)^{2}}{2}+\frac{p_{1}}{\rho_{1}}\right]=\frac{I_{0}}{\rho_{1}\left(D-u_{1 n}\right)}-\Delta L}
\end{gathered}
$$

where, $\Delta L$ is dissociation and ionization energy, determined by the ionization rate $\alpha_{i}$ 


$$
\Delta L=L_{d}+\alpha_{i} L_{i}
$$

$L_{d}$ and $L_{i}$ are dissociation energy and ionization energy, respectively.

In the paper, we take the gas besides the LSD wave as polytropic gas, and the state parameters meet the relationship $p_{i}=n_{i} k T_{i}$ and $e_{i}=p_{i} / \rho_{i}\left(\gamma_{i}-1\right)$, where, $i=1,2$. The particle number density front and after wave are related by

$$
\frac{n_{2}}{n_{1}}=\left(1+\alpha_{i}\right) N \frac{\rho_{2}}{\rho_{1}}
$$

where, $N$ is the number of atoms contained in the gas molecule.

The analysis above suggests that determining the plasma ionization rate is the linchpin to construe the impact of dissociation and ionization. Assuming the plasma ionization is Equilibrium, satisfies the Saha equation

$$
\frac{\alpha_{i}^{2}}{1-\alpha_{i}^{2}}=\frac{2.4 \times 10^{-4}}{p_{2}} T_{2}^{2.5} \exp \left(-\frac{L_{i}}{k_{B} T_{2}}\right)
$$

So, the relationship between the ionization rate and the pressure and temperature is established. Thus, in the case of known the parameters before LSD wave, only one added condition is needed to determine the parameters behind the wave, i.e. Jouguet conditions

$$
D=u_{2}+c
$$

where, $c_{2}$ is the speed of sound behind the LSD wave

$$
c_{2}^{2}=\gamma_{2} p_{2} / \rho_{2}
$$

\section{Performance of Laser Propulsion}

Utilize one dimension assumption, constant flux laser induced wave structure and the characteristic lines of the flow behind LSD are shown in Fig 2 . The laser pulse is turned off at the point of $\mathrm{A}$, and the LSD wave is terminated at the same time. $\mathrm{AB}$ is the intersection line of the rarefaction wave regions issued from point $\mathrm{O}$ and point $\mathrm{A}$, respectively. The state parameters on $\mathrm{AB}$, meet the both characteristic relations of the two regions. So the expressions of $\mathrm{AB}$ is of the form

$$
\frac{x}{t}=\left[\frac{\gamma_{2}+1}{\left(\gamma_{2}-1\right)}\left(D-u_{2}\right) \cdot\left(\frac{t}{\tau_{p}}\right)^{-2 \frac{\gamma_{2}-1}{\gamma_{2}+1}}+\left(\frac{\gamma_{2}+1}{\gamma_{2}-1} u_{2}-\frac{2 D}{\gamma_{2}-1}\right)\right]
$$

OB can be expressed as 


$$
x=\left(D-\frac{\gamma_{2}+1}{2} u_{2}\right) t
$$

The time $\tau_{b}$, corresponding to $\mathrm{B}$ is

$$
\tau_{B}=\tau_{p}\left[\frac{2 D-\left(\gamma_{2}+1\right) u_{2}}{2\left(D-u_{2}\right)}\right]^{-\frac{\gamma_{2}+1}{2\left(\gamma_{2}-1\right)}}
$$

Therefore, the time that the rarefaction wave reaches the target surface is evaluated as follows

$$
\tau_{S}=2 \tau_{B}=2 \tau_{p}\left[\frac{2 D-\left(\gamma_{2}+1\right) u_{2}}{2\left(D-u_{2}\right)}\right]^{-\frac{\gamma_{2}+1}{2\left(\gamma_{2}-1\right)}}
$$

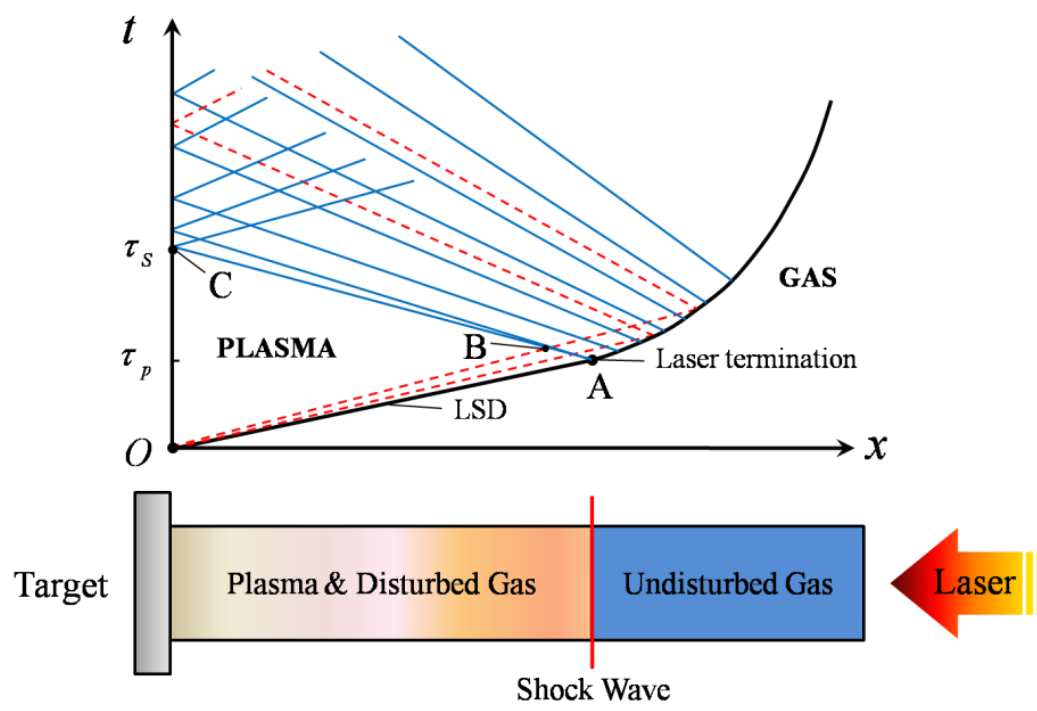

Fig 2 Characteristic lines of the flow behind LSD induced by constant flux laser.

When $t<\tau_{\mathrm{S}}$, the pressure on the target is constant, according to Taylor wave's relationship ${ }^{[3]}$, the pressure $p_{S}$ is of the form

$$
p_{S}=p_{2}\left[\frac{2 D-\left(\gamma_{2}+1\right) u_{2}}{2\left(D-u_{2}\right)}\right]^{\frac{2 \gamma_{2}}{\gamma_{2}-1}}
$$

When $t>\tau_{S}$, spherical explosive detonation wave model is used to describe the change of surface pressure $p_{R}{ }^{[4][6]}$

$$
p_{R}=p_{S}\left(t / \tau_{S}\right)^{-6 / 5}
$$

Therefore, the total impulse $\sigma_{I}$ obtained on the target and the Momentum-Coupling Coefficient $C_{m}$ are of the form as follows 


$$
\begin{gathered}
\sigma_{I}=\int_{0}^{\tau_{S}} p_{S} d t+\int_{\tau_{S}}^{\tau_{t}}\left(p_{R}-p_{1}\right) d t=p_{S} \tau_{S}+5 p_{S} \tau_{S}^{6 / 5}\left(\tau_{S}{ }^{-1 / 5}-\tau_{t}^{-1 / 5}\right)-p_{1}\left(\tau_{t}-\tau_{S}\right) \\
C_{m}=\frac{\sigma_{I}}{I \tau_{p}}
\end{gathered}
$$

where, $\tau_{t}$ is the time when $p_{R}$ is decreased to $P_{1}$.

\section{Results and Discussion}

In the environment of air, consider the physical processes of LSD wave formed above the target surface, and evaluate the impulse coupling performance. Using one-dimensional assumption, the impact of dissociation and ionization to the parameters LSD wave is calculated. What's more, the variations of momentum-coupling coefficient with laser flux intensity and ambient pressure are discussed.

The parameters involved in the calculations are shown in Table 1.

\section{Table 1. Parameters used in the calculation}

\begin{tabular}{c|c|c|c|c}
\hline \hline Component & Molecular weight & Volume ratio $(\%)$ & $L_{d}(\mathrm{~J} / \mathrm{mol})$ & $L_{i}(\mathrm{~J} / \mathrm{mol})$ \\
\hline $\mathrm{N}_{2}$ & 28 & 78 & $9.46 \times 10^{5}$ & $2.80 \times 10^{6}$ \\
$\mathrm{O}_{2}$ & 32 & 21 & $4.98 \times 10^{5}$ & $2.63 \times 10^{6}$ \\
$\mathrm{Ar}$ & 40 & 1 & - & $1.52 \times 10^{6}$ \\
\hline \hline
\end{tabular}

\subsection{Propagation of LSD and the Flow Parameters Behind}

The propagation velocity of LSD is described in Fig. 3, and the results of Raizer's model and the experimental data are also showed in the figure. According to literature [2], Raizer model evaluates the LSD speed $D_{R}$ and surface pressure $p_{R S}$ as follows

$$
\begin{gathered}
D_{R}=\left[2\left(\gamma_{2}^{2}-1\right) I_{0} / \rho_{1}\right]^{1 / 3} \\
p_{R S}=\left[\left(\gamma_{2}+1\right) / 2 \gamma_{2}\right]^{2 \gamma_{2} /\left(\gamma_{2}-1\right)} \cdot \rho_{1} D_{R}^{2} /\left(\gamma_{2}+1\right)
\end{gathered}
$$

In Fig. 3, the solid line denotes the results taken account of dissociation and ionization effects, and the dashed line means the Raizer's model. It's obvious that our model agrees better with the experimental data, particularly when the laser intensity is not so strong. Because the intensity is smaller, the effect of the plasma radiation is less significant. With the increase of the laser intensity, the deviation of the modified model and Raizer's model enlarges gradually. This is because the gas ionization rate is increased as the laser intensity growing; the proportion of the total laser energy used for ionization gradually becomes larger. 


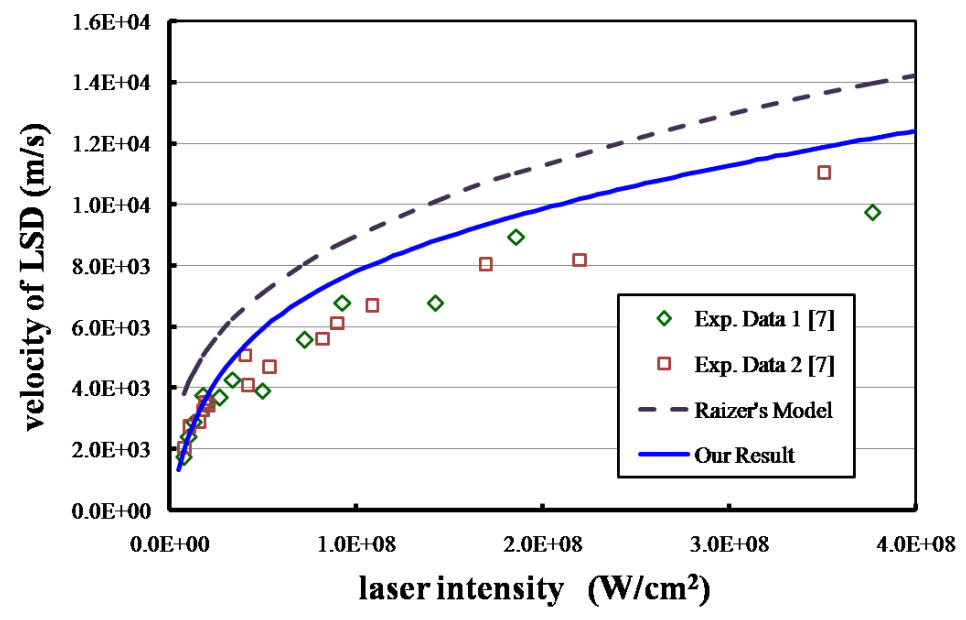

Fig 3 Variation of velocity of LSD with laser flux intensity.

Fig 4 shows the variation of the surface pressure on the target with laser flux intensity, when the environment pressure is $1 \mathrm{~atm}$. The divergent of the experimental data is visible. The graph suggests that Raizer's model fits the upper of the experimental results well, while the result of modified model approximates to the average value better. When the laser intensity is small, the deviation of the two models is evident; with the increase of the laser intensity the difference is reduced step by step. The reason lays to that, as the light intensity mounting up, the percentage of dissociation and ionization energy upswings first and then declines and can be ignored finally; while the particle number density increases gradually and tends to be stable.

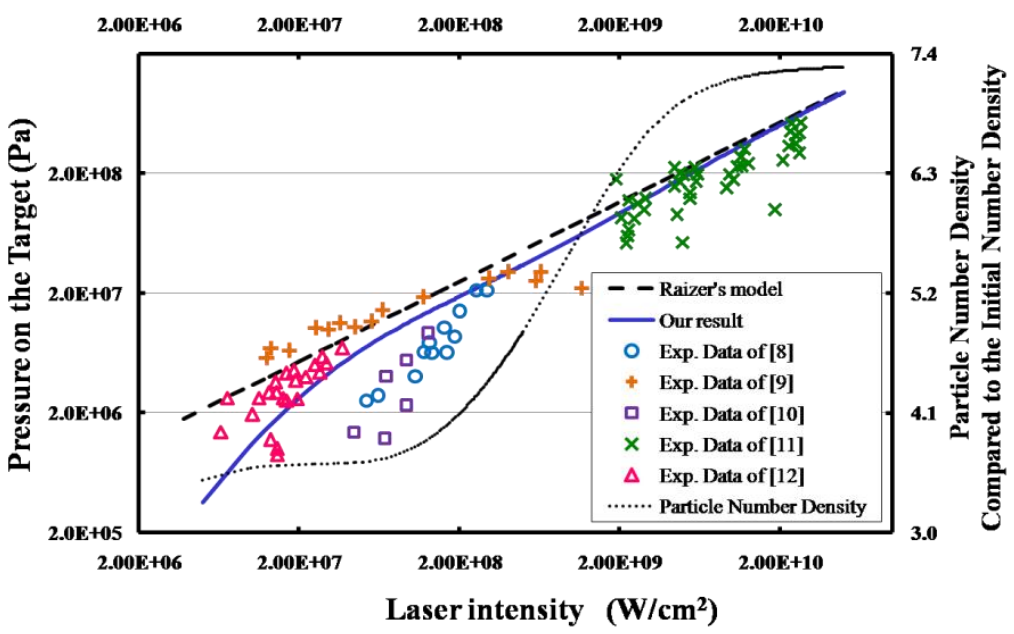

Fig 4 Variation of the surface pressure with laser flux intensity, ( $\left.p_{1}=1 \mathrm{~atm}\right)$.

\subsection{Characteristics of Momentum-Coupling Coefficient}

The relationship between momentum-coupling coefficient and laser intensity is shown in Fig. 5, appended with the result obtained from Raizer's model and the experimental results. If analyze just from the graph, both model fit the Exp. Data poorly, although the modified model is better, relatively. The main reasons for this phenomenon mainly include three aspects. Firstly, the computational model using a one-dimensional approximation, do not consider the sparse effect of two-dimensional; when the spot radius is small or pulse duration is long, the error will be notable. Secondly, without considering the energy loss of reflection, transmission and radiation, the effective absorption of laser energy is less than the incident energy. Thirdly, the temporal distribution of the laser used in the calculation is rectangular, 
which has great discrepancy in intensity distribution compared with the actual laser. The low intensity part of the actual laser pulse will weaken the impulse coupling performance by inducing rarefaction wave. To account for these effects, a weakening factor would introduce to the calculation. The dashed line in Fig. 5 shows the result when the weakening factor is 0.5 , which agrees with the experimental results well. What needs to point out particularly, compared with Raizer's model our model can describe the trend of the variation of momentum-coupling coefficient with laser flux intensity accurately, i.e. first increasing and then decreasing. The result indicates that dissociation and ionization are important reasons for the appearance of momentum-coupling coefficient extremum ${ }^{[14]}$.

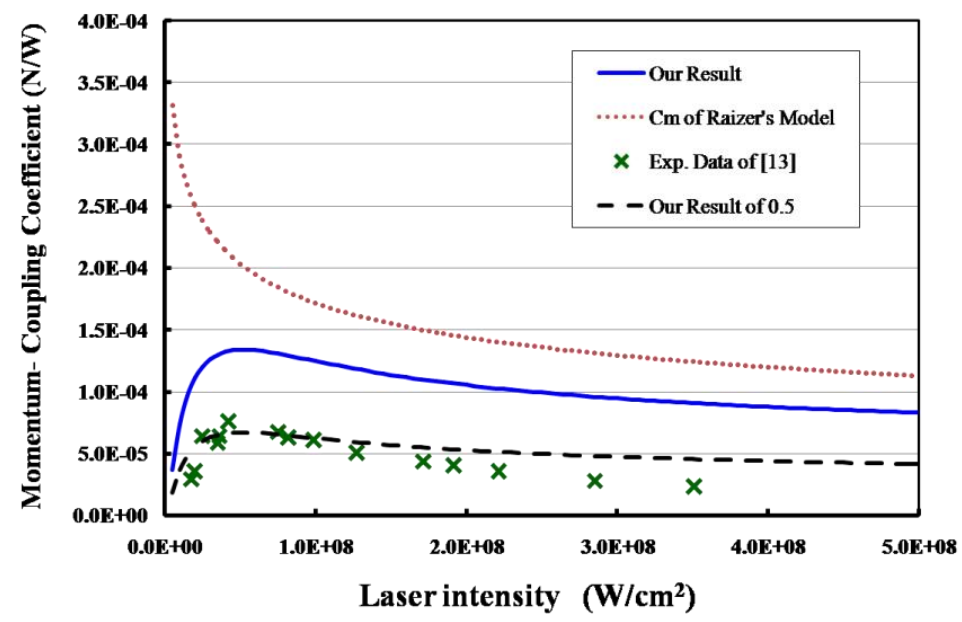

Fig 5 Variation of momentum-coupling coefficient with laser flux intensity, $\left(p_{1}=1\right.$ atm $)$.

Fig 6 depicts Variations of momentum-coupling coefficient with laser flux intensity under different ambient pressure. Firstly, taking a look at the whole picture, with the increase of laser intensity, all lines change with the same tendency of first increasing then decreasing. The comparison of different curves suggested that the optimum intensity corresponding to the largest momentum-coupling coefficient rises with the growth of the ambient pressure, but the maximum coefficient is approximately equal to 130N/MW. In addition, the momentumcoupling coefficient is larger when the ambient pressure is greater at the same laser intensity, if the light intensity is large enough.

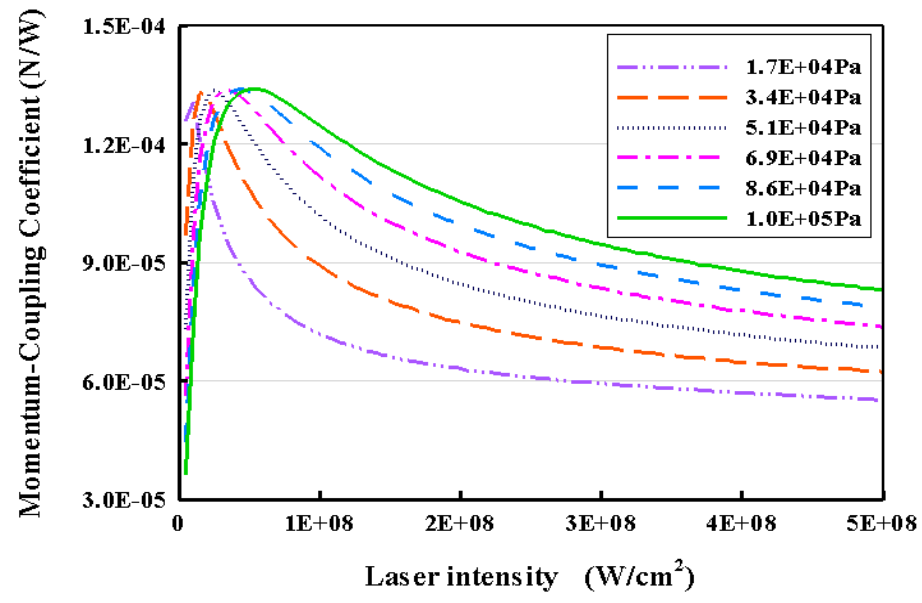

Fig 6 Variations of momentum-coupling coefficient with laser flux intensity under different ambient pressure. 


\section{Conclusions}

In the process of LSD development and dissemination, effects of dissociation and ionization are mainly manifested in two aspects: one is the variation of particle number; the second is the conversion of the laser energy. Considering the influence in the mass, momentum and energy conservation relations, a modification of the classical Raizer's LSD model is presented in this paper. By calculating the modified model, the LSD wave parameters are obtained. Compared to Raizer's model, our results conform to the experimental data much better. Based on the modified model, with one-dimensional assumption, the momentum-coupling properties of laser propulsion are predicted, and the effects of the laser intensity and ambient pressure on impulse coupling performance are also discussed. The main conclusions of this paper are:

(1) Because of the energy dissipation of dissociation and ionization, the LSD propagation velocity is decreased.

(2) Particle number density is increased by dissociation and ionization, which eases the pressure reduction due to energy consumption of dissociation and ionization.

(3) With the increasing of laser intensity, the momentum-coupling coefficient increases firstly and then decreases, dissociation and ionization are important causes of the appearance of impulse coupling coefficient extremum.

The continuation of this work should be carried on from three aspects. Firstly, detailed analysis of two-dimensional effect on propulsion performance is worth to do, by considering the influence of lateral rarefaction on target surface pressure. Secondly, according to the actual shape of the laser pulse, using numerical simulation method, determining the flow entrance boundary conditions by the modified LSD model, a simulation of flow between the LSD wave and the target surface could be done, and then analyzes the propulsion performance of the actual waveform. The third, considering the laser propagation and Inverse Bremsstrahlung absorption in the plasma region, analyze the influence of wave-length to the propulsion qualities.

\section{References}

[1]. Phipps C., Birkan M., Bohn W., et al. "Review: Laser-Ablation Propulsion", Journal of Propulsion and Power, vol. 26(4), 2010: pp. 609-637.

[2]. Raizer Y. P., "Laser-induced discharge phenomena", Consultants Bureau, New York, 1977, pp. 193-198.

[3]. Pirri A. N., "Theory of momentum transfer to a surface with a high-power laser", Phys. Fluids, vol. 16(9), 1973, pp.1435-1440.

[4]. Reilly J. P., Ballantyne A., Woodroffe J. A. "Modeling of Momentum Transfer to a Surface by Laser-Supported Absorption Waves", AIAA Journal, vol. 17(10), 1979, pp. 1098-1105.

[5]. Shimamura K., Hatai K., Kawamura K., et al. "Internal structure of laser supported detonation waves by two-wavelength Mach-Zehnder interferometer ", Journal of Applied Physics, vol. 109, 2011, pp. 084910.

[6]. Sedov L. I., "Similarity and Dimensional Methods in Mechanics", Academic Press, New York, 1959, pp. 9-12. 
[7]. Maher W. E., Hall R. B., Johnson R. R., "Experimental study of ignition and propagation of lasersupported detonation waves", J. Appl. Phys., vol. 45, 1974, pp. 2138.

[8]. Bettehe L. R., Schrieapf J. T., Stegean R. L., "Impulse reaction resulting from the in-air irradiation of alumium by a pulsed CO2 laser", J. Appl. Phys., vol. 44, 1973, pp. 40794085.

[9]. Boiko V. A., Danilychev V. A., Duvanov D. N., et al. "Measurement of gasdynamic pressure on a target subjected to CO2 laser radiation", Sov. J. Quantum Electron, vol. 7, 1977, pp. 465-468.

[10]. Ready J. F., "Laser-produced shocks and their relation to material damage", IEEE Journal of Quantum Electronics, vol. 14, 1978, pp. 79.

[11]. King H. H., Ludvig K. A., Nenna T. L., "Measurements of stresses induced in targets by a 30 nsec 1.06 micron laser pulse in air", Genetal Research Corp. Rept., 1983, CRB1182 .

[12]. Holmes B. S., "Pulsed laser thermal mechanical damage study", AFWL-TR-80-31, vol. $1,1981$.

[13]. Rudder R. R., "Momentum transfer to Solid target discs by pulsed one-sicrom radiation" AFWL-TR-73-273, 1973.

[14]. Sinko J. E., Sasoh A., "Review of CO2 Laser Ablation Propulsion with Polyoxymethylene", International Journal of Aerospace Innovations, vol. 3(2), 2011, pp. 93-129. 